\title{
Identification of possible ion-drag induced neutral instability in the lower thermosphere over Svalbard
}

\author{
C. M. Hall ${ }^{1}$ and T. Aso ${ }^{2}$ \\ ${ }^{1}$ Troms $\phi$ Geophysical Observatory, University of Troms $\phi$, Norway \\ ${ }^{2}$ National Institute of Polar Research, Tokyo, Japan
}

(Received May 1, 2000; Revised August 1, 2000; Accepted August 29, 2000)

\begin{abstract}
The EISCAT Svalbard Radar (ESR) has been used to obtain ion velocities in the lower thermosphere. By using beam swinging and assuming homogeneity and stationarity of the plasma, first approximations to the electric field have been deduced, and thus the thermospheric neutral wind has been estimated. From these derived parameters, we have estimated the gradient Richardson Number. Although many assumptions must be made, there is an indication that electrodynamics is able to contribute to enhancement or even production of neutral-air turbulence in the lower thermosphere. Finally, we outline a proposal for an analogy to the Reynolds Number, but reflecting the relative importance's of the contribution of ion-drag to the neutral dynamics and the kinematic viscosity.
\end{abstract}

\section{Introduction}

The European Incoherent Scatter (EISCAT) Svalbard Radar (ESR) is located at $78^{\circ} \mathrm{N}, 16^{\circ} \mathrm{E}$ (Wannberg et al., 1997). Hall and Aso (1999) used this system to obtain the zonal component of ion velocity in the upper mesosphere and lower thermosphere by directing the antenna at low elevation to the west. These measurements were only partly successful due to transmitter problems curtailing the experiment prematurely. A second experiment was performed starting at $18 \mathrm{UT}$ on 17th and ending at 18 UT on the 18th March 1999. The experimental features are given in Table 1. A fundamental difference between this experiment and that of Hall and Aso (1999) was that the antenna was directed alternately west and south with 15-minute dwell time in each direction. This strategy, designed to obtain both zonal and meridional tidal components, precluded the gravity-wave spectral slope study performed previously. Otherwise, the results were similar as can be seen from Fig. 1, which shows the zonal ion wind at a selection of heights: during hours of darkness, the incoherent scatter signal is poor and there is considerable spread in the results; during daylight hours, the signal gives more credible results and the tidal dynamics is quite obvious. The results shown here are intended to be representative of the horizontal (positive eastward) ion wind on the assumption that the vertical component is relatively very small: the line-of-sight (Doppler) speeds are simply divided by - cosine $\left(45^{\circ}\right)$. The results for each dwell period were averaged. The "error bars" show the 1-sigma variation obtained from the averaging process.

We have used these results to estimate the electric field, subject to some assumptions. There is a trade-off here: a low elevation reduces the need for assuming negligible vertical

Copy right (C) The Society of Geomagnetism and Earth, Planetary and Space Sciences (SGEPSS); The Seismological Society of Japan; The Volcanological Society of Japan; The Geodetic Society of Japan; The Japanese Society for Planetary Sciences. ion velocity, but at the same time increases the requirement for homogeneity since the scattering volumes are further apart. Also, short dwell times reduce the need for stationarity, but similarly reduce the averaging time. The electric field may be estimated by utilising:

$$
\begin{aligned}
& E_{y}=-u_{\text {ion }} \cdot|\mathbf{B}| \\
& E_{x}=+v_{\text {ion }} \cdot|\mathbf{B}|
\end{aligned}
$$

where $E_{x, y}$ are the zonal and meridional components respectively of the electric field $\mathbf{E}$ in geomagnetic coordinates; $u_{\text {ion }}$ and $v_{\text {ion }}$ are the zonal and meridional components respectively of the ion velocity $\mathbf{v} ; \mathbf{B}$ is the magnetic field, which we obtain from the International Geomagnetic Reference Field (IGRF) (http://nssdc.gsfc.nasa.gov/space/ model/models/igrf.html) The inclination of the geomagnetic field is approximately $80^{\circ}$ at Longyearbyen such that $E_{x, y}$ lie approximately in the geographic horizontal plane. The results are shown in Fig. 2.

\section{Atmospheric Stability-Theory}

Hitherto, we have presented characteristics of the ionospheric plasma. Let us now explore how this information may be used to explore the neutral atmosphere, in particular the state of dynamic stability as affected by the ion-dynamics.

In the lower thermosphere, neutral temperature increases rapidly with height, this characteristic being the origin of the very term "thermosphere". Because of this, the likelihood of convective instability is small, however, dynamic instability may still be present. These kinds of instability may be parameterised by the gradient Richardson Number, $R i$ :

$$
R i=\frac{\omega_{B}^{2}}{\left(\frac{\mathrm{dU}}{\mathrm{d} z}\right)^{2}}
$$

where $\omega_{B}$ is the Brunt-Väisälä frequency, $U$ is the hori- 

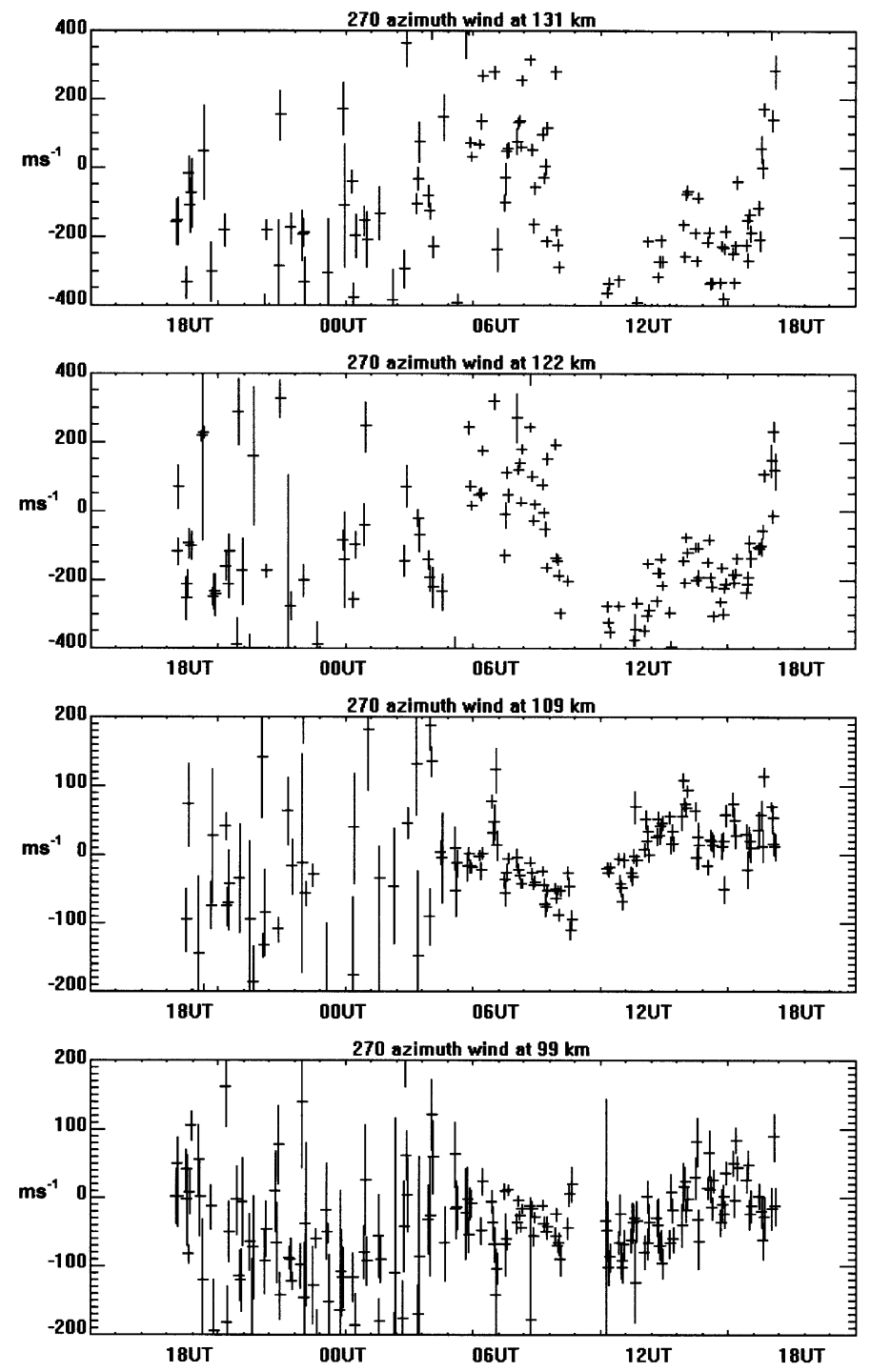

Fig. 1. Examples of upper mesosphere / lower thermosphere zonal ion winds determined by EISCAT Svalbard radar. The experiment started at 18 UT on the 17th March 1999 and ran for 24 hours.

Table 1. EISCAT Svalbard radar features.

\begin{tabular}{ll}
\hline Parameter & Value \\
\hline latitude & $78^{\circ} \mathrm{N}$ \\
longitude & $16^{\circ} \mathrm{E}$ \\
operating frequency & $500 \mathrm{MHz}$ \\
peak power & $1 \mathrm{MW}$ \\
height resolution & $1.5 \mathrm{~km}$ \\
time resolution (this study) & $15 \mathrm{~min}$ \\
elevation (this study) & $45^{\circ}$ \\
azimuth (this study) & $270^{\circ}$ for $15 \mathrm{~min}$ \\
& $180^{\circ}$ for $15 \mathrm{~min}$ \\
antenna & $32 \mathrm{~m}$ dish \\
\hline
\end{tabular}

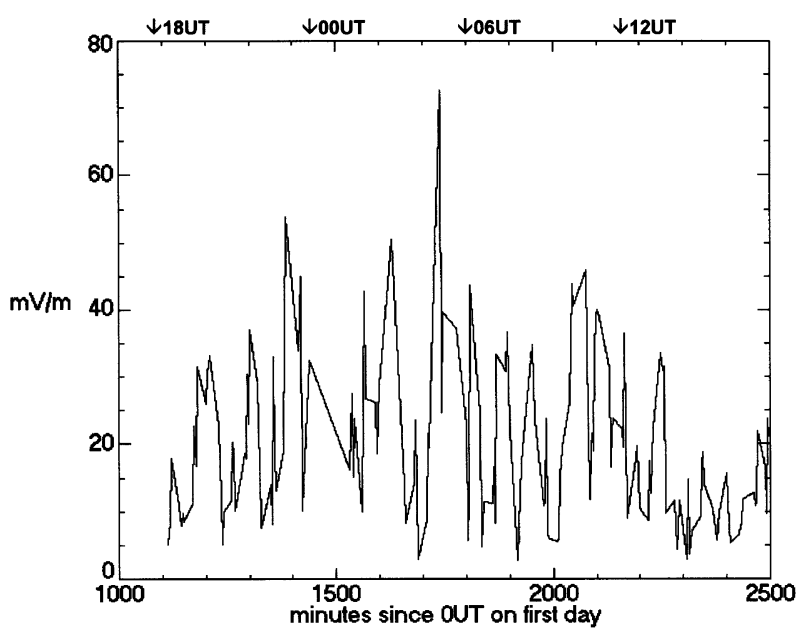

Fig. 2. Total electric field derived from the F-region ion velocity and corresponding to the $24 \mathrm{~h}$ period starting at 18 UT on 17th March 1999. 
zontal neutral wind and $z$ is height. When $R i$ is negative the atmosphere is convectively unstable ( $\omega_{B}$ is imaginary); when $0<R i<0.25$ there is a dynamic instability and turbulence may be expected; existing turbulence may be just maintained when $R i=0.4$; the atmosphere will be stable for $R i>1$. These various conditions have been much discussed in the literature and should not be taken as de facto (e.g. Weinstock, 1978; Roper and Brosnahan, 1997).

If we assume that the electric field may be mapped down the geomagnetic field, estimates of $E$ made in the F-region (where the ion-neutral collision frequency is sufficiently small that the ion motion may be considered independent of that of the neutral gas), may be utilised to estimate the neutral motion in the underlying E-region (i.e. the lower thermosphere) by (e.g. Fujii et al., 1998):

$$
\mathbf{U}_{\perp} \approx \mathbf{V}_{\perp}-\frac{e}{m_{i}} \frac{\mathbf{E}_{\perp}}{v_{i n}}
$$

where $\mathbf{V}_{\perp}, \mathbf{U}_{\perp}$ and $\mathbf{E}_{\perp}$ are the ion and neutral winds and the electric field respectively in the plane perpendicular to the magnetic field $\mathbf{B}, e$ is the electronic charge, $m_{i}$ is the ion mass and $v_{i n}$ is the ion-neutral collision frequency. Here, it is assumed that $e B \ll m_{i} v_{i n}$ or equivalently $v_{i n} \gg \Omega_{i}$, the ion gyro frequency. If we note that, at high latitude, $\mathbf{B}$ is directed almost vertically downwards, $\mathbf{U}$ and $\mathbf{U}_{\perp}$ may be approximated. Therefore, assuming $\mathbf{E}$ is constant with height, we may differentiate (3) with respect to height and obtain the neutral wind shear:

$$
\frac{\mathrm{d} \mathbf{U}_{\perp}}{\mathrm{d} z} \approx \frac{\mathrm{d} \mathbf{V}_{\perp}}{\mathrm{d} z}+\frac{e|\mathbf{E}|}{v_{i n}^{2} m_{i}} \frac{\mathrm{d} v_{i n}}{\mathrm{~d} z}
$$

Typically: $v_{i n}=2.6 \times 10^{-15}\left(n_{n}+n_{i}\right) M_{n}^{-1 / 2} \operatorname{per} \operatorname{second}\left(n_{n, i}\right.$ being the neutral and ion number densities, respectively and at the altitudes we are concerned with $\left.n_{n} \gg n_{i}\right) ; M_{n}=30.5$ amu, the neutral molecular weight, this value being typical for the lower thermosphere and below; $e=1.6 \times 10^{-19}$ coulomb, the electronic charge; and $m_{i}=30 \times 1.7 \times 10^{-27}$ $\mathrm{kg}$, the ion mass. It may appear that the neutral motion is controlled by that of the ions, but in fact the ions will tend to follow the neutral motion, but be "dragged out of course" by the electrodynamics (viz. Eq. (3)). The derivation of the neutral wind by the method described here is subject to three substantial assumptions: (i) stationarity of the E-field in the F-region, since the ion vector velocities in E- and F-regions are not determined instantaneously; (ii) homogeneity of the E-field since we use a beam-swinging method in this particular experiment; (iii) invariability (with height) of the E-field between the F- and E-region measurements. The last assumption, in particular may be open to question on the highlatitude dayside since the observation is made at or near the cusp. However, given these assumptions, we see that by observing the ion motion at consecutive heights, along with estimating the electric field, we are able to deduce the neutral wind and shear, and hence $R i$. It should be stressed that the observation described here represents a pilot experiment to examine the feasibility of investigating dynamic instability using incoherent scatter radar, and furthermore at high latitude; in future measurements, the E-field determination should be improved.

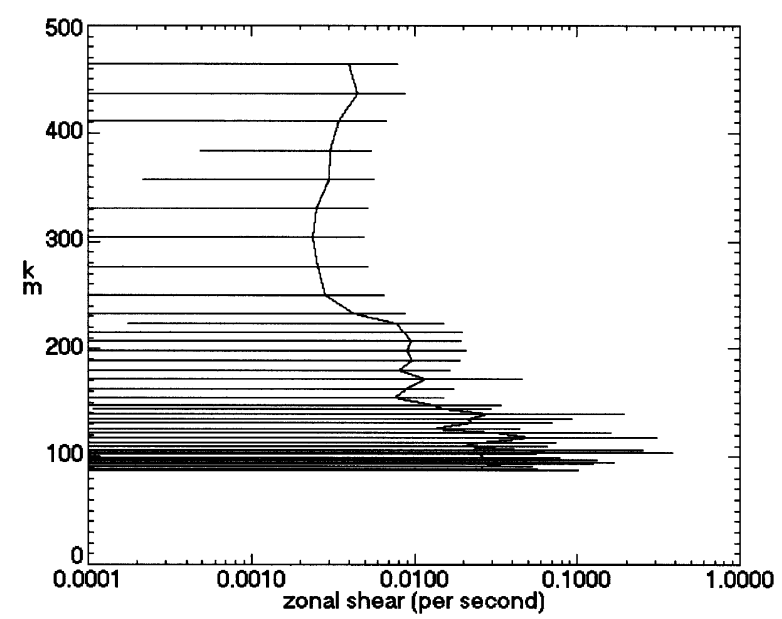

Fig. 3. Absolute values of average ion-drag induced neutral wind shear. The "error" bars indicate the variability suggested by the variability (standard deviation) in the electric field. The derivation of these values is valid for the lower thermosphere and below; the higher altitude values, included for completeness, should be regarded with suspicion.

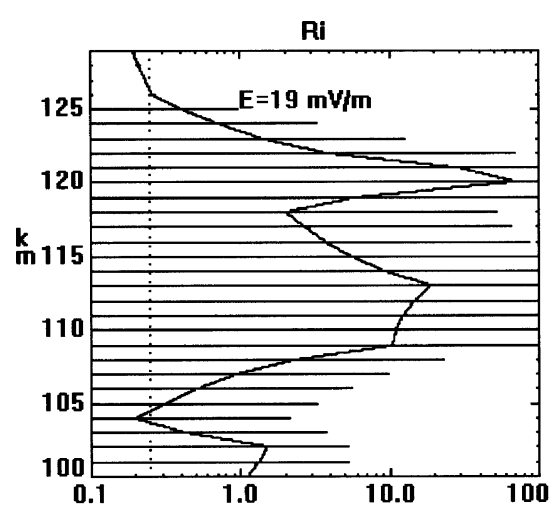

Fig. 4. Average gradient Richardson number due to neutral wind shear induced by ion-drag. The vertical dotted line indicates the instability condition $R i<0.25$.

In addition to $R i$, the Reynolds Number, $R e$, is often used to identify likelihood for turbulent flow. We have

$$
R e=\text { velocity-scale } \times \text { length scale/kinematic viscosity. }
$$

When the length-scale is that of the largest eddies, i.e. the "outer scale", $L_{B}$, and the velocity scale is the eddy velocity, Re may be thought of as the ratio of eddy to molecular diffusivities. For example, the turbopause may be defined as the height at which $R e=1$. While it is common to associate turbulent flows with high $\operatorname{Re}(\gg 1)$, one may also invert this concept such that when $R e<1$ any turbulence ceases to exist because viscous damping does not allow the eddies to exist. We may compare the ion-drag over a vertical interval to the neutral kinematic viscosity, thus proposing an analogy to $R e$ which reflects the relative importance of electrodynamics (on the neutral motion) to viscosity. Identifying the term containing $\mathbf{E}$ in (4) as the electrodynamic contribution, one can make the analogy

$$
\aleph \equiv \frac{\text { (ion-drag induced shear } \times \text { length }) \times \text { length }}{\text { kinematic viscosity }}
$$




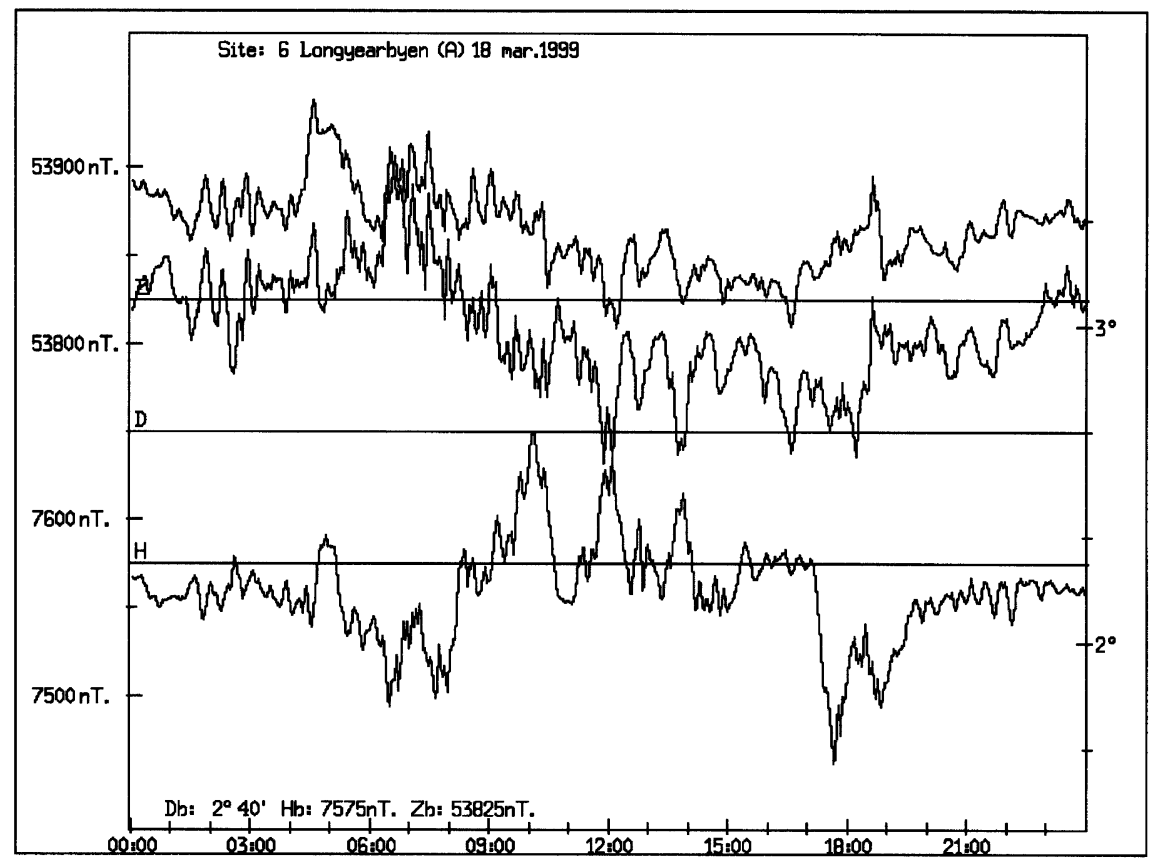

Fig. 5. Co-located magnetometer record for 18th March 1999. The traces represent, from top to bottom, Z, D and H components respectively.

where $\aleph$ is the "electrodynamics analogy to $R e$ ". One way of enumerating this quantity could be

$$
\aleph \equiv\left|\frac{e|\mathbf{E}|}{v_{i n}^{2} m_{i}} \frac{\mathrm{d} v_{i n}}{\mathrm{~d} z} \cdot \frac{L_{B}^{2}}{v}\right| .
$$

Here we have used $L_{B}$ (e.g. Blix et al., 1990) as the length scale and the ion-drag induced shear multiplied by $L_{B}$ as the velocity scale. Other ways, utilising time scales are also possible; we shall not pursue this further here, but rather concentrate on estimation of $R i$. Nevertheless, in future estimation of turbulence, profiles of $\aleph$ should be included since whenever $\aleph<1$ turbulence will be inhibited anyway by viscosity irrespective of $R i$; Hall (2000) explains this in detail. It should be recognised that $\aleph$ is not the same as $R e$, it includes the ion-drag component only, $R e$ itself ceasing to exceed unity at heights above the turbopause, by definition.

\section{Atmospheric Stability-Measurements}

Before proceeding to combine the incoherent scatter measurements summarised in the Introduction with the theory described above, it should be stressed that we are performing a pilot study only to assess the feasibility of determining $R i$ and $\aleph$. To simplify the calculation, we will work with dayaverage profiles. The average electric field was found to be $19 \mathrm{mVm}^{-1}$ with a standard deviation of $11 \mathrm{mVm}^{-1}$. Since sources of uncertainty are numerous and encompass both experimental errors and assumptions, we shall only show variability as indicated by the standard deviation. As stated earlier, the E-field measurement, and more specifically the assertion that this represents the E-region value depends on three important assumptions: stationarity during the beamswinging, homogeneity over the swing, and validity of fieldline mapping. Furthermore, due to the low elevation of the radar beam, the E-region measurements are not on the same field line as the F-region measurements. Again, this study should be regarded as an investigation of a new technique rather than a de facto measurement. Nevertheless, we note that in addition to a moderately quiet geomagnetic field (discussed later) the Kp index varied around 2 for the daylight times of the measurent period. We conclude that the velocity measurements were facilitated by photoionisation, which in turn is likely to be fairly stationary over the beam swing cycle and horizontally homogeneous over the geographic area covered.

In order to obtain the kind of stability information described above, the MSISE90 model atmosphere (Hedin, 1991) will be employed for nominal values of neutral number density and temperature necessary for estimating $v, v_{i n}$ and $\omega_{B}$. The average ion-drag induced zonal neutral shear is shown in Fig. 3. Here we show the absolute values of the average shear with "error bars" indicating the 1-sigma variability (which in turn reflect the variability in the electric field. The derivation of these values applies to where the cyclotron frequency is smaller than the collision frequency, i.e. the lower thermosphere and below; the higher altitude values are illustrative only. Similar results were obtained for the meridional component. We see that $20-30 \mathrm{~ms}^{-1} \mathrm{~km}^{-1}$ shears were common in the lower thermosphere. Combining the total shear with $\omega_{B}$ derived from MSISE90, we arrive at the corresponding $R i$ profile, shown Fig. 4 . The dotted line indicates $R i=0.25$. Above around $123 \mathrm{~km}$ and in a region centred on $104 \mathrm{~km}$ the shears are sufficient for enhancement of existing dynamic instability (i.e. turbulence) if not destabilisation of a previously neutral atmosphere. However, kinematic viscosity is likely to suppress turbulence above the turbopause $(R e<1)$, or more specifically, wherever $\aleph<1$. Thus, the higher altitude instances of small $R i$ are unlikely to produce turbulence, whereas the lower instances may indeed indicate turbulent regions. Since the error bars actually 
indicate variability, one can envisage that on occasions of substantial electric field $\left(|\mathbf{E}|\right.$ often exceeded $\left.40 \mathrm{mVm}^{-1}\right)$ the instability was considerably greater. The co-located magnetometer data is shown, for completeness, in Fig. 5. The electric field is determined by the ion motion in the F-region and is not necessarily correlated with geomagnetic variation. The geomagnetic variability is influenced mainly by E-region currents, which, in turn, depend not only on the E-field but also on the conductivity. As we see, geomagnetic activity was modest, consistent with ionisation being caused almost exclusively by insolation as we saw from the post-dawn increase in signal-to-noise ratio.

\section{Conclusion}

We have presented ion-velocity data from lower thermosphere heights above Svalbard. With assumptions as to stationarity and homogeneity of the wind field in the F-region, we have made estimates of the electric field, and therefore the ion-drag. Next, we have postulated that the ion-drag induced neutral wind shear may be used to indicate gradient Richardson numbers and hence evidence for dynamic instability. Finally, we propose an analogy to the Reynolds number: electrodynamics-induced neutral motion divided by kinematic viscosity. At heights where this metric is greater than unity and $R i<0.25$ one might anticipate neutral turbulence to occur. Indeed such a height region is in evidence, even in our day-averaging of our data. We conclude it likely that neutral-air turbulence may be either generated, or at least enhanced, by ion-drag in the lower thermosphere during periods of high electric field.
Acknowledgments. We are indebted to the Director and staff of EISCAT for operating the facility and supplying the data. EISCAT is an International Association supported by Finland (SA), France (CNRS), the Federal Republic of Germany (MPG), Japan (NIPR), Norway (NFR), Sweden (NFR) and the United Kingdom (PPARC). In particular, we wish to thank Tony van Eyken for provision of analysis, telescience, and data transfer. Thanks also go to the referees of this paper.

\section{References}

Blix, T. A., E. V. Thrane, and Ø. Andreassen, In situ measurements of the fine-scale structure and turbulence in the mesosphere and lower thermosphere by means of electrostatic positive ion probes, J. Geophys. Res., 95, 5533-5548, 1990.

Fujii, R., S. Nozawa, S. C. Buchart, N. Matuura, and A. Brekke, The motion of ions in the auroral ionosphere, J. Geophys. Res., 103, 20,685-20,695, 1998.

Hall, C. M., An analogy to the Reynolds number for the neutral gas component of a weak plasma, Ann. Geophys., 2000 (in press).

Hall, C. M. and T. Aso, Mesospheric velocities and buoyancy subrange spectral slopes determined over Svalbard by ESR, Geophys. Res. Lett., 26, 1685-1688, 1999.

Hedin, A. E., Extension of the MSIS thermosphere model into the middle and lower atmosphere, J. Geophys. Res., 96, 1159-1172, 1991.

Roper, R. G. and J. W. Brosnahan, Imaging Doppler interferometry and the measurement of atmospheric turbulence, Radio Sci., 32, 1137-1148, 1997.

Wannberg, U. G., I. Wolf, L.-G. Vanhainen, K. Koskenniemi, J. Röttger, M. Postila, J. Markkanen, R. Jacobsen, A. Stenberg, R. Larsen, S. Eliassen, S. Heck, and A. Huuskonen, The EISCAT Svalbard Radar, a case study in modern incoherent scatter radar system design, Radio Sci., 32, 22832307, 1997.

Weinstock, J., Vertical turbulent diffusion in a stably stratified fluid, J. Atmos. Sci., 35, 1022-1027, 1978.

C. M. Hall (e-mail: chris.hall@phys.uit.no) and T. Aso 